\title{
HILBERT-SCHMIDT REPRESENTATIONS OF GROUPS
}

\section{LARRY BAGGETT ${ }^{1}$}

Introduction. A completely continuous representation of a locally compact group $G$ is a unitary representation $T$ of $G$ for which the operator $T_{b}$ is completely continuous whenever $b$ is an element of the group $C^{*}$-algebra $C^{*}(G)$. We have also that, with respect to the norm operator topology, $T_{[L(G)]}$ is dense in the set $T_{\left[L^{1}(G)\right]}$ which in turn is

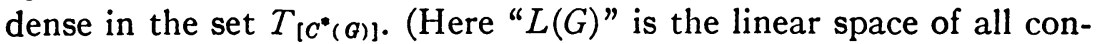
tinuous complex-valued functions on $G$ with compact support.) Further, it is known that, if $T$ is an irreducible completely continuous representation of $G$, the set $T_{\left[C^{*}(G)\right]}$ is precisely the algebra of all completely continuous operators on the Hilbert space $H(T)$. (See [4].) Investigation of completely continuous representations has been fruitful because, first of all, such representations are more easily analyzed, and secondly, a large class of groups (CCR-groups [2]) satisfies the condition that each of their irreducible unitary representations is a completely continuous representation.

The algebra $A$ of all Hilbert-Schmidt operators on a Hilbert space is dense in the algebra of all completely continuous operators on that space. Therefore, it was natural to inquire what simplifications arise if one requires that a completely continuous representation of a group $G$ should map either the set $L(G)$ into the space of all HilbertSchmidt operators or the set $L^{1}(G)$ into the space of all HilbertSchmidt operators. In this paper we show that too much simplification occurs in the latter case. In fact, if $T_{f}$ is a Hilbert-Schmidt operator whenever $f$ is an element of $L^{1}(G)$, then $T$ must be finite dimensional. (See the theorem below.)

In the first case mentioned above, the simplifications, if there are any, are more elusive, and we shall return to this question at the end of the article.

Definition 1. Let $G$ be a locally compact group. By a unitary representation of $G$ we mean a homomorphism $T$ of $G$ into the group of unitary operators on a Hilbert space $H(T)$ which is continuous with respect to the weak-operator topology. (See [3].)

We single out this definition from representation theory because, in the course of the proof below, the continuity condition here is heavily exploited.

Received by the editors July 15, 1968.

1 This research was supported by NSF Grant GP-6869. 
Definition 2. Let $H$ be a Hilbert space and let $\left[v_{a}\right]$ be an orthonormal basis for $H$. An operator $T$ on $H$ is called a Hilbert-Schmidt operator if $\sum_{a}\left\|T\left(v_{a}\right)\right\|^{2}$ is finite. (See [1].)

THEOREM. Let $T$ be a unitary representation of a locally compact group $G$ for which $T_{f}$ is a Hilbert-Schmidt operator whenever $f$ is an element of $L^{1}(G)$. Then $T$ is finite dimensional.

Proof. Assume that $\left[v_{n}\right]$ is an infinite orthonormal sequence in $H(T)$. With this assumption, we shall define a sequence $\left[\left(n_{k}, w_{k}, U_{k}, f_{k}\right)\right]$ of quadruples, where $\left[n_{k}\right]$ is an increasing sequence of positive integers, $w_{k}=v_{\left(n_{k}\right)},\left[U_{k}\right]$ is a sequence of neighborhoods of the identity $e$ in $G$, and $f_{k}$ is a multiple of the characteristic function $\chi_{\left(U_{k}\right)}$ of the neighborhood $U_{k}$. We will then show that $f=\sum_{k=1}^{\infty} f_{k}$ is an element of $L^{1}(G)$ such that $T_{f}$ is not a Hilbert-Schmidt operator.

Thus let $\epsilon$ be a positive number which is less than one, and, for each positive integer $k$, define $a_{k}$ to equal $(1 / k)^{1 / 2}-(1 /(k+1))^{1 / 2}$.

Let $n_{1}=1, w_{1}=v_{1}, U_{1}$ be a neighborhood of $e$ with finite measure such that, for each element $y$ of $U_{1}$,

$$
r e\left(T_{y}\left(w_{1}\right), w_{1}\right)>\epsilon,
$$

and $f_{1}=\left(a_{1} / \lambda\left(U_{1}\right)\right) \chi_{\left(U_{1}\right)}$. (We denote Haar measure by $\lambda$.)

Now if $\left(n_{1}, w_{1}, U_{1}, f_{1}\right) \cdots\left(n_{k}, w_{k}, U_{k}, f_{k}\right)$ have been defined, we proceed as follows to define $\left(n_{(k+1)}, w_{(k+1)}, U_{(k+1)}, f_{(k+1)}\right)$. Let $n_{(k+1)}$ be an integer which is greater than $n_{k}$, and for which

$$
\left\|T_{\left[\Sigma_{i=1}^{k} f_{i}\right]}\left(v_{n}\right)\right\|<\epsilon /(k+1)
$$

for each integer $n \geqq n_{(k+1)}$. Such an integer exists because $T_{\left[\Sigma_{i-1}^{*} f_{i}\right]}$ is by assumption a Hilbert-Schmidt operator and consequently is completely continuous.

Define $w_{(k+1)}$ to be $v_{\left(n_{(k+1)}\right)}$; define $U_{(k+1)}$ to be the intersection of $U_{k}$ with the set of all elements $y$ in $G$ such that

$$
\operatorname{re}\left(T_{y}\left(w_{(k+1)}\right), w_{(k+1)}\right)>\epsilon ;
$$

finally define $f_{(k+1)}$ to be $\left(a_{(k+1)} / \lambda\left(U_{(k+1)}\right)\right) \chi_{\left(U_{(k+1)}\right)}$. This completes our inductive definition.

REMARK 1. For each positive integer $k,\left\|f_{k}\right\|_{1}=a_{k}$. Hence $f=\sum_{k=1}^{\infty} f_{k}$ is an element of $L^{1}(G)$, and $\|f\|_{1}=1$. Notice also that $\sum_{k=n}^{\infty} a_{k}$ $=(1 /(n+1))^{1 / 2}$.

REMARK 2. If $k$ and $n$ are positive integers such that $k>n$, and if $y$ is an element of $U_{k}$, then $y$ is also an element of $U_{n}$ and $r e\left(T_{y}\left(w_{n}\right), w_{n}\right)$ $>\epsilon$. 
We now compute to see that $T_{f}$, where $f=\sum_{k=1}^{\infty} f_{k}$, is not a HilbertSchmidt operator on $H(T)$. Thus let $\left[v_{a}\right]$ be an extension of $\left[v_{n}\right]$ to an orthonormal basis for $H(T)$. Then:

$$
\begin{aligned}
& \sum_{a}\left\|T_{f}\left(v_{a}\right)\right\|^{2} \geqq \sum_{n}\left\|T_{f}\left(v_{n}\right)\right\|^{2} \geqq \sum_{n}\left\|T_{f}\left(w_{n}\right)\right\|^{2} \\
& \vdots \geqq \sum_{n}\left|\left(T_{f}\left(w_{n}\right), w_{n}\right)\right|^{2}=\sum_{n}\left|\left(T_{\left[\sum_{k=1}^{\infty} f_{k}\right]}\left(w_{n}\right), w_{n}\right)\right|^{2} \\
& =\sum_{n}\left|\left(T_{\left[\sum_{k=1}^{n-1} f_{k}\right]}\left(w_{n}\right), w_{n}\right)+\left(T_{\left[\Sigma_{k=n}^{\infty} f_{k}\right]}\left(w_{n}\right), w_{n}\right)\right|^{2} \\
& \geqq \sum_{n}\left|\left[\left|\left(T_{\left[\sum_{k=n}^{\infty} f_{k}\right]}\left(w_{n}\right), w_{n}\right)\right|-\left|\left(T_{\left[\sum_{k=1}^{n-1} f_{k}\right]}\left(w_{n}\right), w_{n}\right)\right|\right]^{2}\right| \\
& =\sum_{n} \mid\left[\left|\left(T_{\left[\Sigma_{k-n}^{\infty} f_{k}\right]}\left(w_{n}\right), w_{n}\right)\right|^{2}+\left|\left(T_{\left[\Sigma_{k=1}^{n-1} f_{k}\right]}\left(w_{n}\right), w_{n}\right)\right|^{2}\right. \\
& \left.-2\left|\left(T_{\left[\sum_{k-n}^{\infty} f_{k}\right]}\left(w_{n}\right), w_{n}\right)\right|\left|\left(T_{\left[\sum_{k=1}^{n-1} f_{k}\right]}\left(w_{n}\right), w_{n}\right)\right|\right] \mid \\
& \geqq \sum_{n}\left[\left|\left(T_{\left[\sum_{k=n}^{\infty} f_{k}\right]}\left(w_{n}\right), w_{n}\right)\right|^{2}+\left|\left(T_{\left[\sum_{k=1}^{n-1} f_{k}\right]}\left(w_{n}\right), w_{n}\right)\right|^{2}\right. \\
& \left.-2\left|\left(T_{\left[\sum_{k=n}^{\infty} f_{k}\right]}\left(w_{n}\right), w_{n}\right)\right|\left|\left(T_{\left[\Sigma_{k=1}^{n-1} f_{k}\right]}\left(w_{n}\right), w_{n}\right)\right|\right] \\
& \geqq \sum_{n}\left[\left|\sum_{k=n}^{\infty}\left(T_{\left(\xi_{k}\right)}\left(w_{n}\right), w_{n}\right)\right|^{2}-\left\|T_{\left[\Sigma_{k=1}^{n-1} f_{k}\right]}\left(w_{n}\right)\right\|^{2}\right. \\
& \left.-2\left|\left(T_{\left[\Sigma_{k=n}^{\infty} f_{k}\right]}\left(w_{n}\right), w_{n}\right)\right|\left\|T_{\left[\Sigma_{k=1}^{n-1} f_{k}\right]}\left(w_{n}\right)\right\|\right] \\
& \geqq \sum_{n}\left[\left[r e\left[\sum_{k=n}^{\infty}\left(T_{\left(\sigma_{k}\right)}\left(w_{n}\right), w_{n}\right)\right]\right]^{2}-\epsilon^{2} / n^{2}-2 \sum_{k=n}^{\infty}\left\|f_{k}\right\|_{1} \epsilon / n\right] \\
& =\sum_{n}\left[\left[\sum_{k=n}^{\infty} r e\left(T_{\left(\xi_{k}\right)}\left(w_{n}\right), w_{n}\right)\right]^{2}-\epsilon^{2} / n^{2}-2\left(\epsilon / n(n+1)^{1 / 2}\right)\right]
\end{aligned}
$$

(see Remark 1)

$$
\begin{aligned}
& =\sum_{n}\left[\left[\sum_{k=n}^{\infty} r e \int_{G} f_{k}(y)\left(T_{y}\left(w_{n}\right), w_{n}\right) d \lambda(y)\right]^{2}\right. \\
& \left.-\epsilon^{2} / n^{2}-2\left(\epsilon / n(n+1)^{1 / 2}\right)\right] \\
& =\sum_{n}\left[\left[\sum_{k=n}^{\infty}\left(a_{k} / \lambda\left(U_{k}\right)\right) r e \int_{\left(U_{k}\right)}\left(T_{y}\left(w_{n}\right), w_{n}\right) d \lambda(y)\right]^{2}\right. \\
& \geqq \sum_{n}\left[\epsilon^{2}\left[\sum_{k=n}^{\infty} a_{k}\right]^{2}-\epsilon^{2} / n^{2}-2\left(\epsilon / n(n+1)^{1 / 2}\right)\right]
\end{aligned}
$$


(see Remark 2)

$$
=\sum_{n}\left[\epsilon^{2} /(n+1)-\epsilon^{2} / n^{2}-2\left(\epsilon / n(n+1)^{1 / 2}\right)\right]=\infty,
$$

and this shows that $T_{f}$ is not a Hilbert-Schmidt operator.

COROLlaRY 1. If $T$ is an infinite dimensional unitary representation of a locally compact group $G$, then there must exist an element $f$ of $L^{1}(G)$ for which the operator $T_{f}$ has infinite dimensional range.

Definition 3. Let $H$ be a Hilbert space. An operator $T$ on $H$ is said to have finite trace if there exist Hilbert-Schmidt operators $S$ and $S^{\prime}$ on $H$ such that $T=S S^{\prime}$. (See [1].)

COROLLARY 2. If a unitary representation $T$ of a locally compact group $G$ satisfies the condition that $T_{f}$ has finite trace whenever $f$ is an element of $L^{1}(G)$, then $T$ is finite dimensional.

Proof. This follows from the theorem since the product of HilbertSchmidt operators is again a Hilbert-Schmidt operator.

COROLlaRY 3. If a unitary representation $T$ of a locally compact group $G$ satisfies $T_{b}$ is a Hilbert-Schmidt operator whenever $b$ is an element of $C^{*}(G)$, then $T$ is finite dimensional.

Of course this is well known in case $T$ is irreducible. If $T$ is not irreducible, the result is less obvious since one can construct a normclosed subalgebra $A$ of the algebra $B(H)$ of all bounded linear operators on an infinite dimensional Hilbert space $H$ such that each element $T$ of $A$ has one dimensional range, hence is a Hilbert-Schmidt operator, but for which the union of the ranges of the elements of $A$ is all of $H$. This corollary asserts that such a subalgebra of $B(H)$ cannot occur as the image of a group $C^{*}$-algebra.

We turn now to the question mentioned in the introduction of what happens if we require only that a unitary representation $T$ of a locally compact group $G$ carry each element $f$ of $L(G)$ onto a HilbertSchmidt operator. The following propositions indicate the striking difference between this case and the one dealt with above. They demonstrate the existence of many infinite dimensional unitary representations which map $L(G)$ into the class of Hilbert-Schmidt operators.

Proposition 1. If $G$ is a compact abelian group, $R$ is the left regular representation of $G$, and $f$ is an element of $L(G)$, then $R_{f}$ is a HilbertSchmidt operator on $L^{2}(G)$. 
Proof. If $\left[\chi_{n}\right]$ is the set of all characters on $G$, then $\left[\chi_{n}\right]$ is an orthonormal basis for $L^{2}(G)$. The proposition then follows because $f^{*} \chi_{n}=\left(f, \chi_{n}\right) \chi_{n}$.

Proposition 2. If $T$ is an irreducible unitary representation of a simply connected semisimple Lie group, then $T_{f}$ is a Hilbert-Schmidt operator whenever $f$ is an element of $L(G)$.

This proposition may be deduced from a theorem of HarishChandra. (See Theorem 5 of Representations of a semisimple Lie group. III, Trans. Amer. Math. Soc. 76 (1954), 234-253.)

Proposition 3. Let $G$ be a unimodular group. If $T$ is an irreducible subrepresentation of the left regular representation of $G$, then $T_{f}$ is a Hilbert-Schmidt operator whenever $f$ is an element of $L(G)$.

This result is included in a paper which the author has submitted for publication.

We remark, finally, that results have been obtained concerning unitary representations which map "sufficiently" differentiable functions onto Hilbert-Schmidt operators. (See Theorem 4.2 of Nelson and Stinespring's article Representations of elliptic operators in an enveloping algebra, Amer. J. Math 81 (1959), 547-560.)

The author greatly appreciates the referee's recommending the above article as well as the Harish-Chandra article mentioned in Proposition 2.

\section{BIBLIOGRAPHY}

1. N. Dunford and J. Schwartz, Linear operators. Part II, Interscience, New York, 1963.

2. I. Kaplansky, The structure of certain operator algebras, Trans. Amer. Math. Soc. 70 (1951), 219-225.

3. G. W. Mackey, Notes on group representations, University of Chicago, Chicago, IIl., 1956.

4. M. A. NaImark, Normed rings, GITTL, Moscow, 1956; English transl., Noordhoff, Groningen, 1959.

UNIVERSITY OF COLORADO 